\title{
Impaired Vigilant Attention Partly Accounts for Inhibition Control Deficits After Total Sleep Deprivation and Partial Sleep Restriction
}

\author{
Tianxin Mao ${ }^{1,2}$ \\ David Dinges ${ }^{3}$ \\ Yao Deng $\mathbb{( D}^{1,2}$ \\ Ke Zhao $\mathbb{D}^{2}$ \\ Zijing Yang ${ }^{2,4}$ \\ Hui Lei ${ }^{2}$ \\ Zhuo Fang ${ }^{2}$ \\ Fan Nils Yang ${ }^{2}$ \\ Olga Galli ${ }^{3}$ \\ Namni Goel (D $)^{5}$ \\ Mathias Basner ${ }^{3}$ \\ Hengyi Rao ${ }^{1-3}$
}

'Key Laboratory of Applied Brain and Cognitive Sciences, School of Business and Management, Shanghai International Studies University, Shanghai, People's Republic of China; ${ }^{2}$ Center for Functional Neuroimaging, Department of Neurology, University of Pennsylvania, Philadelphia, PA, USA; ${ }^{3}$ Division of Sleep and Chronobiology, Department of Psychiatry, University of Pennsylvania, Philadelphia, PA, USA; ${ }^{4}$ School of Medicine, Shanghai Jiaotong University, Shanghai, People's Republic of China; ${ }^{5}$ Biological Rhythms Research Laboratory, Department of Psychiatry and Behavioral Sciences, Rush University Medical Center, Chicago, IL, USA

Correspondence: Hengyi Rao Center for Functional Neuroimaging \& Department of Neurology, University of Pennsylvania Perelman School of

Medicine, Room D502, Richards Medical Research Building, 3700 Hamilton Walk, Philadelphia, PA, 19104-4202, USA

Tel +I 215-746-2470

Email hengyi@pennmedicine.upenn.edu
Purpose: Sleep loss impairs a range of neurobehavioral functions, particularly vigilant attention and arousal. However, the detrimental effects of sleep deprivation on inhibition control and its relationship to vigilant attention impairments remain unclear. This study examined the extent to which vigilant attention deficits contribute to inhibition control performance after one night of total sleep deprivation (TSD) and two nights of partial sleep restriction (PSR).

Participants and Methods: We analyzed data from $\mathrm{N}=49$ participants in a one-night of TSD experiment, $\mathrm{N}=16$ participants in a control experiment without sleep loss, and $\mathrm{N}=16$ participants in a two-nights of PSR experiment (time in bed, TIB $=6 \mathrm{~h}$ for each night). Throughout waking periods in each condition, participants completed the psychomotor vigilance test (PVT), which measures vigilant attention, and the Go/No-Go task, which measures inhibition control.

Results: After TSD and PSR, participants displayed significantly slower reaction times (RT) and more lapses in PVT performance, as well as slower Go RT and more errors of omission during the Go/No-Go task. PVT deficits accounted for $18.0 \%$ of the change in Go RT and $12.4 \%$ of the change in errors of omission in the TSD study, and $23.7 \%$ of the change in Go RT and $20.3 \%$ of the change in errors of omission in the PSR study.

Conclusion: Both TSD and PSR impaired inhibition control during the Go/No-Go task, which can be partly accounted for by vigilant attention deficits during the PVT. These findings support the key role of vigilant attention in maintaining overall neurobehavioral function after sleep loss.

Keywords: total sleep deprivation, partial sleep restriction, vigilant attention, inhibition control

\section{Plain Language Summary}

Previous research suggests that vigilant attention deficits may be the general factor underlying cognitive impairments following sleep deprivation. However, the detrimental effect of sleep loss on inhibition control and its relationship to vigilant attention impairment after sleep deprivation remains unclear. Here we examined the extent to which performance deficits in the Go/No-Go task are explained by vigilant attention impairments after TSD and PSR. The results demonstrate that both TSD and PSR significantly impair inhibition control, which can be partly explained by vigilant attention deficits. These findings support the fundamental role of vigilant attention in maintaining neurobehavioral function during insufficient sleep.

\section{Introduction}

Sleep accounts for about a third of our time and is an indispensable part of human life. However, with the development of science and technology, and the 
acceleration of the pace of life, the phenomenon of sleep loss has become a significant public health concern. Partial sleep restriction (PSR) and total sleep deprivation (TSD) are two common forms of sleep deprivation. Many previous studies demonstrate that both forms of sleep loss can negatively affect an individual's cognition, mood, and physical health. ${ }^{1-8}$

Inhibition control refers to the ability to choose appropriate responses while suppressing inappropriate and unwanted behavior. It is a core component of executive function and is essential for controlling human behavior. ${ }^{9,10}$ Deficient inhibition control may negatively affect individuals' career goals and social relationships. ${ }^{11}$ Poor inhibition control is generally associated with the attentional deficits shown by attention deficit hyperactivity disorder patients, violence, drug addiction, and suicidal behavior. ${ }^{12}$ One of the most commonly used paradigm for measuring inhibition control is the Go/No-Go task, which requires individuals to respond as quickly as possible to select stimuli while withholding a response to all other stimuli. ${ }^{13}$

Due to the prevalence of sleep loss and the importance of inhibition control, many researchers have explored the effects of sleep disturbance or sleep deprivation on inhibition control. ${ }^{14}$ For example, one previous event-related potentials (ERP) study found that poor sleep affects the speed to inhibit a motor response as well as the intensity of pre-motor inhibitory processes and task-relevant information processing, ${ }^{15}$ while a more recent study found that sleep deprivation impairs memory control with sleepdeprived participants showing more unsuccessful suppression of intrusions of emotionally negative and neutral scenes than rested participants. ${ }^{16}$ However, the effects of both total and partial sleep deprivation on inhibition control are not consistent. In TSD experiments, some studies have found that inhibition control is significantly diminished from baseline after TSD, ${ }^{17-22}$ while others found no significant changes. ${ }^{23}$ Similarly, some studies using PSR have found that inhibition control is decreased significantly after sleep loss. ${ }^{24-28}$ However, some other studies found no significant changes in inhibition control after PSR. $^{29,30}$

Several factors may contribute to the inconsistencies in the literature. For example, some sleep deprivation studies were conducted outside of the laboratory, ${ }^{24,27,28}$ and many confounding variables (eg, room temperature, noise, interactions with family members) may not have been well controlled. Moreover, different task paradigms and lengths of sleep deprivation or sleep restriction protocols have been used in previous studies. ${ }^{25-31}$ For example, one ERP study used the Go/No-Go ERP paradigm and found that accuracy of detection of the rarely occurring Go and No-Go stimuli significantly declined after $24 \mathrm{~h}$ and $36 \mathrm{~h}$ of continued wakefulness. Moreover, the amplitudes of both the Go and No-Go P300 components were also significantly reduced after $24 \mathrm{~h}$ and $36 \mathrm{~h}$ of continuous wakefulness, suggesting that sleep deprivation may not have a specific effect on inhibition. ${ }^{32}$ In contrast, another study explored the effect of noise-induced sleep disturbances on inhibition functions in a visual Go/No-Go task and found that decision processes underlying overt responses were less vulnerable to disturbed sleep than inhibition processes. ${ }^{33}$ Since the underlying mechanisms that drive the adverse effects of TSD and PSR may not be the same,$^{34}$ more rigorous laboratory studies are needed to investigate the effects of both TSD and PSR on inhibition control.

In addition to inhibition control, many studies have consistently demonstrated the detrimental effects of sleep loss on a range of other cognitive domains, such as attention and working memory. Although the sensitivity of cognitive tasks to sleep deprivation varies widely, ${ }^{35}$ previous studies have shown that both TSD and PSR yield significant impairments in vigilant attention. ${ }^{2,7,8,36-39}$ Because vigilant attention is essential for high-level cognition functions and is most susceptible to sleep deprivation, it has been proposed that vigilant attention may be the fundamental factor that explains much of the variance in other cognitive deficits following sleep deprivation. ${ }^{40}$ This hypothesis highlights the importance of vigilant attention and suggests that high-level cognitive functions will be impaired if an individual is not sufficiently vigilant to perform a task. ${ }^{40-42}$ Although this hypothesis has existed for years, few studies have explicitly examined the relationships between vigilant attention and performance on the inhibition control tasks under conditions of sleep loss.

The aims of the present study were to examine the effects of both TSD and PSR on inhibition control and determine whether impairments in inhibition control induced by TSD and PSR can be explained by deficits in vigilant attention, testing the Vigilance Hypothesis. ${ }^{33}$ The data for the current analyses were derived from two separate sleep deprivation experiments. Experiment 1 examined the effects of one night of TSD on inhibition control and the relationship between inhibition control and vigilant attention following TSD. We hypothesized 
that (1) after a night of TSD, inhibition control and vigilant attention would be significantly impaired when compared to baseline sleep; and that (2) changes in inhibition control would be correlated with changes in vigilant attention after TSD. Experiment 2 investigated the effects of two nights of PSR on inhibition control and the relationship between inhibition control and vigilant attention. Similarly, we hypothesized that (1) compared with baseline, the level of individual inhibition control and vigilant attention would decrease under PSR, with greater deficits observed after two nights of PSR; and that (2) deficits in individual inhibition control would also be correlated with deficits in vigilant attention after PSR.

\section{Materials and Methods}

\section{Experiment I: Effects of TSD on} Inhibition Control

\section{Participants}

A total of 65 healthy adults participated in the experiment. Forty-nine of these adults (mean age $34.1 \pm 8.7$ years, 18 females, all right-handed) participated in the TSD condition, and 16 (mean age $35.7 \pm 8.6$ years, seven females, all right-handed) participated in the control group study. All participants were nonsmokers and had no acute or chronic medical and psychological conditions. As assessed by circadian rhythm questionnaires ${ }^{43}$ and actigraphy, all participants had a regular bedtime (22:00-00:00) and wake time (06:00-09:00) with a total sleep time duration of 6.5 to 8 hours, and no habitual napping. Individuals were excluded if they participated in shift work, traveled across time zones, or had irregular sleep-wake routines in the 60 days before the study. Individuals with sleep disorders were excluded by a night of laboratory polysomnography and oximetry measurements. Bedtimes and wake times of enrolled participants were assessed by actigraphy, sleep logs, and time-stamped call-ins during the week before and after the laboratory stay. No caffeine, alcohol, tobacco, or medications (except oral contraceptives) were permitted in the week before and during the study. This study was conducted in accordance with the Declaration of Helsinki and was approved by the Institutional Review Board of the University of Pennsylvania. All participants provided written informed consent before enrollment and were compensated for their time.

\section{Procedure}

The laboratory protocol lasted five consecutive days and four nights, but for this report, only data from the first three days and two nights were used. Some other data from the study have been published in earlier articles. $^{44,45}$ All participants were required to stay in the controlled laboratory room at the Center for Human Phenomic Science at the Hospital of the University of Pennsylvania, where they were monitored by trained staff throughout the study. They were required to arrive at the laboratory on the afternoon of Day 1 . They were provided with a 9-hour time in bed (TIB) sleep opportunity (from $21: 30$ to $06: 30$ ) on the first night to allow for habituation to an unfamiliar sleep environment. On the second night, participants in the TSD group were asked to remain awake throughout the whole night, while participants in the control group were provided with an 8-hour TIB opportunity for sleep. This was followed by two consecutive nights of recovery sleep opportunity, which are not included in the present report. All participants completed cognitive testing every 2 hours during wakefulness. Specifically, on the Day 2 and Night 2, the TSD group participants were tested at 10:00, 12:00, 14:00, 16:00, 18:00, 20:00, 22:00, 0:00, $02: 00,04: 00$, and 06:00, and the control group was tested at 10:00, 12:00, 14:00, 16:00, 18:00, 20:00, and 22:00. On Day 3, the TSD group participants were tested at 10:00, $12: 00,14: 00$, and 18:00, and the control group was tested at 10:00, 12:00, 14:00, 16:00, 18:00, 20:00, and 22:00. The specific experimental protocol is graphically shown in Figure 1A. In order to compare with the results from Experiment 2, we selected the average values of the three test bouts at 10:00, 14:00 and 18:00 as the analysis index.

\section{The Psychomotor Vigilance Test (PVT)}

The PVT is a highly sensitive and widely used test for assessing vigilant attention in sleep deprivation studies. ${ }^{37,46}$ During the test, participants were asked to maintain their attention on a red-outlined rectangle in the center of a black computer screen and press the space keyboard in response to a cue (millisecond counter) that is presented at random (ranging from every 2 to 10 seconds). The millisecond counter stops after the participant presses the button and stays on the screen for an extra second to allow a participant to see his/her last reaction time. We used the standard 10-minute PVT for the study. It requires participants to respond as quickly and accurately as possible, but to avoid false starts (premature responses). ${ }^{47}$ Not responding to 
A

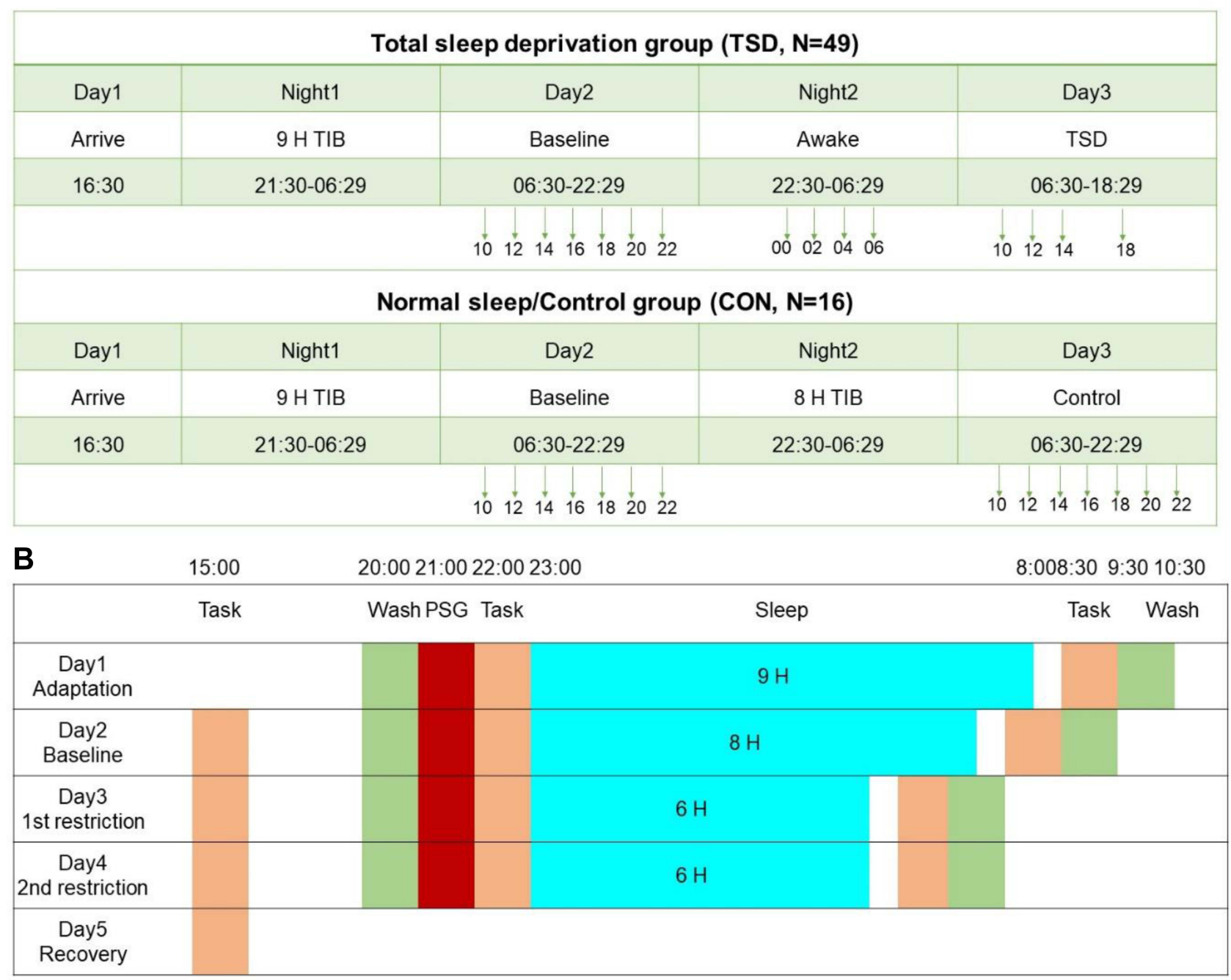

Figure I (A) Experimental protocol for experiment I (total sleep deprivation group and normal sleep/control group); (B) Experimental protocol for experiment 2 (partial sleep restriction).

the PVT numeric light stimulus within 30 seconds is considered as a timeout and responding when no stimulus is present is considered as a false start. When a false start or a timeout occurs, the corresponding text appears on the screen for 1 second before the end of the trial. Median reaction time, the average reaction time, and the number of lapses (defined as any RT longer than $500 \mathrm{~ms}$ ) were obtained from the PVT performance. Longer reaction times and greater lapses were indicative of decreased vigilant attention.

\section{The Go/No-Go Task}

The Go/No-Go task is the most common paradigm for measuring inhibition control. At the beginning of the test, a fixation point appeared on the screen (presented for $1000 \mathrm{~ms}$ ), followed by a stimulus of " $\mathrm{x}$ " or "+" (presented for $100 \mathrm{~ms}$ ). Participants were asked to press the "space" key immediately after seeing the "+", but not to respond to the " $x$ ". The maximum allowed reaction time window in which participants could respond was $1500 \mathrm{~ms}$. There was a total of 60 trials for this task, of which the Go stimulus (ie, the "+" stimulus that participants should respond to) accounted for $75 \%$, and the No-Go stimulus (ie, the " $x$ " stimulus that participants should not react to) accounted for $25 \%$. Participants saw the following instructions: "Please focus on the central square, and press space to respond to ' + ' and do not respond to ' $\mathrm{x}$ '. Please respond quickly and correctly". Each participant practiced the cognitive task before the experiment to ensure he/she fully understood the instructions. Errors of commission (No-Go errors), errors of omission (Go 
errors), and the average response time to the Go stimulus (Go RT) were obtained from the Go/No-Go task performance. More errors of commission and omission and slower Go RT were indicative of impaired inhibition control.

\section{Data Analyses}

For the TSD study, a paired sample $t$-test was used to compare vigilant attention and inhibition control performance between the baseline and following sleep deprivation. Correlation analysis, the Benjamini-Hochberg False Discovery Rate (FDR) correction, and linear regression analyses were used to examine the relationships between the changes in inhibition control and vigilant attention. For the control group, a paired sample $t$-test was used to compare the vigilant attention and inhibition control performance between the baseline and the corresponding day of TSD.

\section{Experiment 2: Effects of PSR on Inhibition Control \\ Participants}

A total of 16 healthy subjects (mean age $19.8 \pm 1.2$ years, 9 males) participated in this experiment. All participants were screened and had no history of physical or mental illness, no habitual use of drug, alcohol, or caffeine, no shift work or travel across time zones, no habitual napping, and had regular sleep-wake schedules. The screening questionnaires included Morning and Evening Questionnaire-19, ${ }^{48,49}$ Pittsburgh Sleep Quality Index ${ }^{50-52}$ Self-rating Anxiety Scale,${ }^{53,54}$ General Health Questionnaire, ${ }^{55,56}$ and Beck Depression Inventory. ${ }^{57}$ Before the study, participants wore an actigraph and completed a sleep log every day for one week. Data from actigraphy and sleep logs showed that on average participants had a bedtime of 23:41 $\pm 17 \mathrm{~min}$, a waking time of 07:38 $\pm 24 \mathrm{~min}$, and sleep duration of $7.95 \pm 0.54$ $\mathrm{h}$. More detailed information for the participants is shown in Table 1. This study was conducted in accordance with the Declaration of Helsinki and was approved by the ethics committee of South China Normal University. All participants carefully read and signed the informed consent after knowing the specific process of the experiment and were compensated after the experiment.

\section{Procedure}

Participants slept in the laboratory for four consecutive nights. The first night was an adaptation night with a TIB sleep opportunity of 9 hours, which allowed
Table I Demographic Information for the Participants in the PSR Experiment

\begin{tabular}{|l|c|c|c|c|}
\hline & Minimum & Maximum & Mean & $\begin{array}{c}\text { Standard } \\
\text { Error }\end{array}$ \\
\hline Age (years) & 18 & 22 & 19.81 & 1.22 \\
BMI (kg/m2) & 18.52 & 23.84 & 20.35 & 1.69 \\
MEQ & 42 & 58 & 53.44 & 4.80 \\
GHQ & 11 & 20 & 18.25 & 2.52 \\
SAS & 29 & 40 & 35.31 & 3.30 \\
PSQI & 0 & 5 & 3.06 & 1.84 \\
BDI & 0 & 5 & 2.44 & 2.06 \\
\hline
\end{tabular}

Abbreviations: MEQ, Morningness-Eveningness Questionnaire-19; GHQ, General Health Questionnaire; SAS, Self-rating Anxiety Scale; PSQI, Pittsburgh Sleep Quality Index; BDI, Beck Depression Inventory.

participants to become familiarized with the sleeping environment. The second night was a baseline night with a TIB sleep opportunity of 8 hours. The third night and fourth nights were two sleep restriction nights, with 6 hours TIB each night. Only data from the last three nights were included in the formal experimental analysis. Participants arrived at the laboratory 3 hours ahead of bedtime every night for experimental preparation (including taking a shower, placing electrodes for polysomnography (PSG), completing cognitive tasks, and filling out questionnaires). The next morning, participants filled out surveys and completed the cognitive tasks. The participants were then allowed to leave the lab and then returned to the laboratory at 15:00 to complete cognitive tasks and questionnaires. They were free for the rest of the day until 3 hours before bedtime, at which point they returned to the laboratory. Mobile phones, pads, and other self-photogenic electronic devices were not allowed during the experiment. The specific experimental protocol is shown in Figure 1B. We used the average of these three measurements (evening, the next morning, the next afternoon) as an analysis indicator.

\section{The Psychomotor Vigilance Test (PVT)}

The PSR study used a slightly different 10-minute PVT task, in which a black triangle (ie, a stimulus) appeared on the computer screen at random times, with the stimulus presenting for $1 \mathrm{~s}$ and the stimulus interval ranging between $2 \mathrm{~s}-10 \mathrm{~s}$. Participants were asked to press the button to respond immediately after seeing the stimulus. Similar to the TSD study, median reaction time, the average reaction time, and the number of lapses were obtained from the PVT performance. 


\section{The Go/No-Go Task}

A slightly different Go/No-Go task was used to measure inhibition control in the PSR study. In this task, a fixation point "+" appeared on the screen, followed by a circle or a square stimulus. The stimulus was presented for $500 \mathrm{ms,}$ and the participants were required to press the " $G$ " key immediately after seeing the circle but were asked not to respond after seeing the square. There was a total of 120 trials for this task, of which Go trials accounted for $75 \%$ and No-Go trials for $25 \%$. Similar to the TSD study, errors of commission (No-Go errors), errors of omission (Go errors), and the average response time to the Go stimulus (Go RT) were obtained from the Go/No-Go task performance.

\section{Data Analysis}

For the PSR study, repeated measurement analyses of variance (ANOVA) were used to compare vigilant attention and inhibition control performance between the baseline day, after one night of PSR, and after two nights of PSR. Correlation analysis, the Benjamini-Hochberg FDR correction, effect size, and linear regression analysis were used to analyze the relationship between the changes in inhibition control and vigilant attention. All the statistical analyses were conducted by SPSS 22.0 software.

\section{Results}

\section{Go/No-Go Results}

\section{Go/No-Go Performance Under TSD Condition}

As shown in Figure 2A, the mean Go RT $(\mathrm{t}(48)=-2.419$, $p=0.019$, Cohen's $\mathrm{d}=0.346)$ and errors of omission ( $\mathrm{t}$ $(48)=-3.545, p<0.001$, Cohen's $d=0.506$ ) significantly increased after one night of TSD compared to the baseline. There was also a marginal increase in errors of commission after TSD relative to baseline ( $\mathrm{t}(48)=-1.949, p=$ 0.057, Cohen's d $=0.278$ ).

\section{Go/No-Go Performance Under Control Condition} As shown in Figure 2B, there were no significant differences in the mean Go RT, errors of omission, and errors of commission between the two test days (all $p>0.05$ ) for the control group without sleep loss.

\section{Go/No-Go Performance Under PSR Condition}

As shown in Figure 2C, there was a significant main effect of PSR for both the Go RT $(F(2,30)=4.042$, $\left.p=0.028, \eta_{\mathrm{p}}^{2}=0.212\right)$ and errors of omission (F (2, $\left.30)=4.599, p=0.018, \eta_{p}^{2}=0.235\right)$, while the main effect on errors of commission was not significant $(F$ $\left.(2,30)=0.482, p=0.623, \eta_{p}^{2}=0.031\right)$. Post-hoc paired $t$-tests showed that the Go RT was significantly increased after both one night of PSR ( $\mathrm{t}(15)=-2.470$, $p=0.026$, Cohen's $d=0.617)$ and two nights of PSR compared to the baseline ( $\mathrm{t}(15)=-2.393, p=0.030$, Cohen's $d=0.598)$. Meanwhile, errors of omission demonstrated a marginal increase after one night of PSR ( $t(15)=-2.052, p=0.058$, Cohen's $d=0.513)$ and a significant increase after two nights $(\mathrm{t}(15)=$ $-2.583, p=0.021$, Cohen's $d=0.646)$. For errors of commission, there was no significant difference between baseline and one or two nights of PSR (all $p>0.05)$.

\section{PVT Results}

\section{PVT Performance Under TSD Condition}

As shown in Figure 3A, the median RT $(\mathrm{t}(48)=-8.479$, $p<0.001$, Cohen's $\mathrm{d}=1.211)$, mean RT $(\mathrm{t}(48)=-3.982$, $p<0.001$, Cohen's $\mathrm{d}=0.569)$, and number of lapses ( $\mathrm{t}$ $(48)=-7.926, p<0.001$, Cohen's $d=1.132)$ on the PVT significantly increased after one night of TSD compared to the baseline.

\section{PVT Performance Under Control Condition}

As shown in Figure 3B, there was no significant difference in the number of lapses between the two test days $(\mathrm{t}(15)=$ $1.638, p=0.12)$. However, the median RT $(\mathrm{t}(15)=3.098$, $p=0.007$, Cohen's $\mathrm{d}=0.774)$ and mean RT $(\mathrm{t}(15)=$ $2.305, p=0.036$, Cohen's $\mathrm{d}=0.576$ ) were significantly reduced on the second test day as compared to the baseline day.

\section{PVT Performance Under PSR Condition}

There were significant main effects of sleep loss on the median RT $\left(\mathrm{F}(2,30)=8.120, p=0.002, \eta_{\mathrm{p}}^{2}=0.351\right)$ and number of lapses $\left(\mathrm{F}(2,30)=9.766, p=0.001, \eta_{\mathrm{p}}^{2}=\right.$ 0.394). However, the main effect of sleep loss on mean RT was not significant $\left(\mathrm{F}(2,30)=2.041, p=0.148, \eta_{\mathrm{p}}^{2}=\right.$ $0.120)$. Post-hoc paired $t$-tests showed that median RT and number of lapses both significantly increased after one night (for median RT, $\mathrm{t}(15)=-3.390, p=0.004$, Cohen's $\mathrm{d}=0.848$; for number of lapses, $\mathrm{t}(15)=$ $-2.807, p=0.013$, Cohen's $d=0.702$ ) or two nights of PSR (for median RT, t $(15)=-3.711, p=0.002$, Cohen's $\mathrm{d}=0.928$; for number of lapses, $(\mathrm{t}(15)=-4.121, p<$ 0.001 , Cohen's $d=1.030$; see Figure $3 \mathrm{C}$ ) as compared to the baseline. 
A

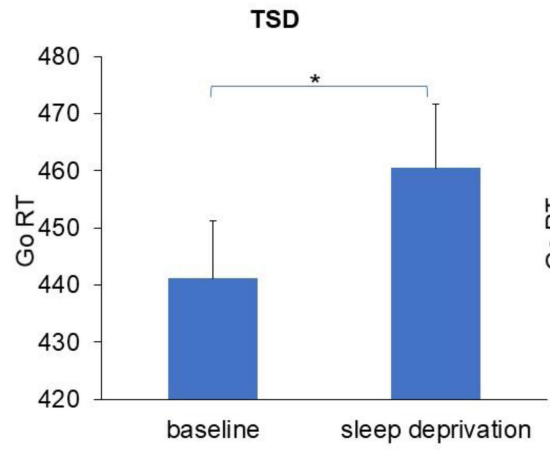

TSD

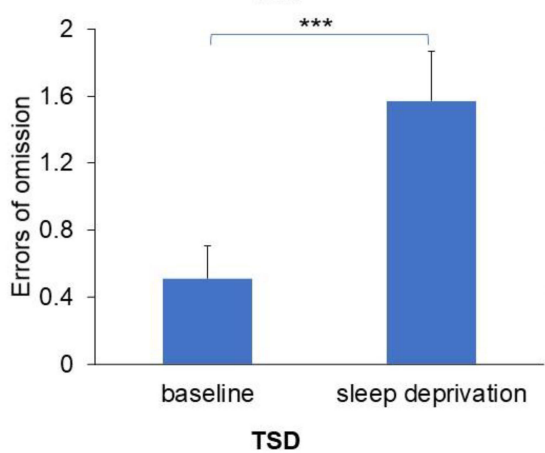

TSD

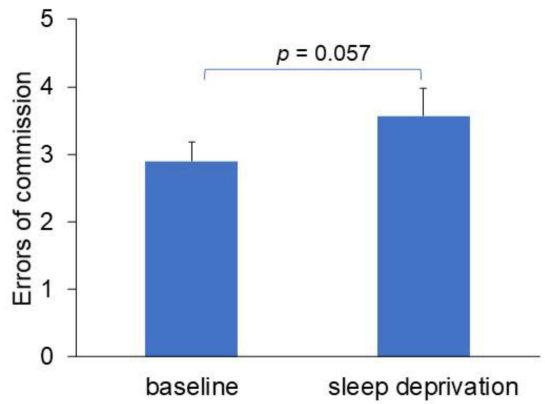

B

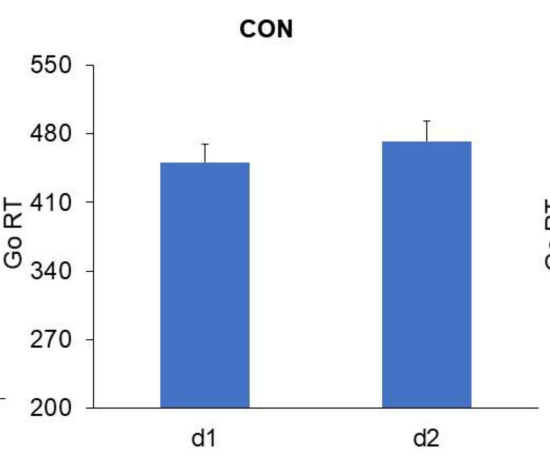

CON

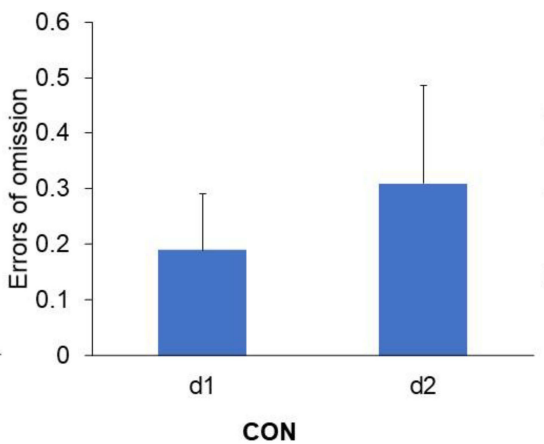

CON

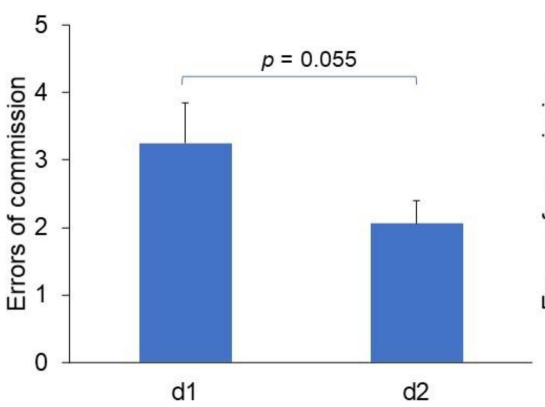

C
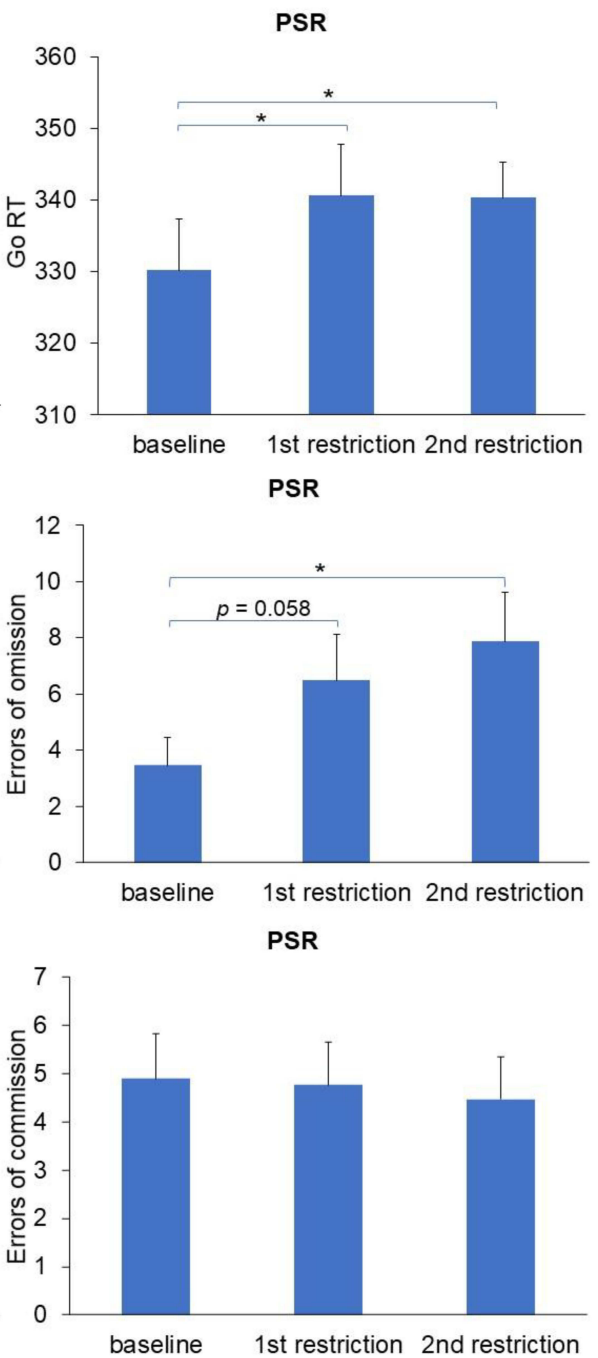

Figure 2 Effects of TSD (Experiment I) and PSR (Experiment 2) on inhibition control (Mean \pm SE) ((A) Go/No-Go performance under TSD condition; (B) Go/No-Go performance under CON condition; (C) Go/No-Go performance under PSR condition).

Notes: ${ }^{*} p<0.05 ; *^{* * *} p<0.001$.

Abbreviations: PSR, partial sleep restriction; TSD, total sleep deprivation; CON, normal sleep (the control study); SE, standard error of the mean; dI, day I in control group (baseline); d2, day 2 in control group (control).

\section{Correlations Between Changes in Gol No-Go Task and Changes in the PVT}

The results from correlation analyses of PVT and Go/Go/NoGo task data for the TSD group are shown in Figure 4A-C and listed in Table 2A. There were significant positive correlations between the $\Delta$ lapses of the PVT and the $\Delta$ errors of omission of the Go/No-Go task (Figure 4A, Pearson $\mathrm{r}=0.352, p=$ 0.013; Spearman rho $=0.430, p=0.002)$, the $\Delta$ median RT of PVT and the $\Delta$ errors of omission of the Go/No-Go task (Figure 4B, Pearson $r=0.290, p=0.043$; Spearman rho $=$ $0.315, p=0.027$ ) after one night of TSD. The changes in the mean RT of PVT also significantly positively correlated with the changes in the errors of omission of the Go/No-Go task (Figure 4C, Pearson $\mathrm{r}=0.425, p=0.002$; Spearman rho $=$ $0.501, p=0.000)$. Then, we performed FDR multiple corrections for all Pearson correlation analysis results using the Benjamini-Hochberg procedure $(\mathrm{BH})$, which is the most commonly used method for FDR correction. ${ }^{58}$ The results after correction were basically consistent with the results before correction. There were significant positive correlations between changes in the PVT mean RT and changes in errors of omission of the Go/No-Go task ( $r=0.42$, FDR $=0.009$ ) after one night of TSD. The $\Delta$ lapses of the PVT were significantly positively correlated with the $\Delta$ errors of omission of 
A
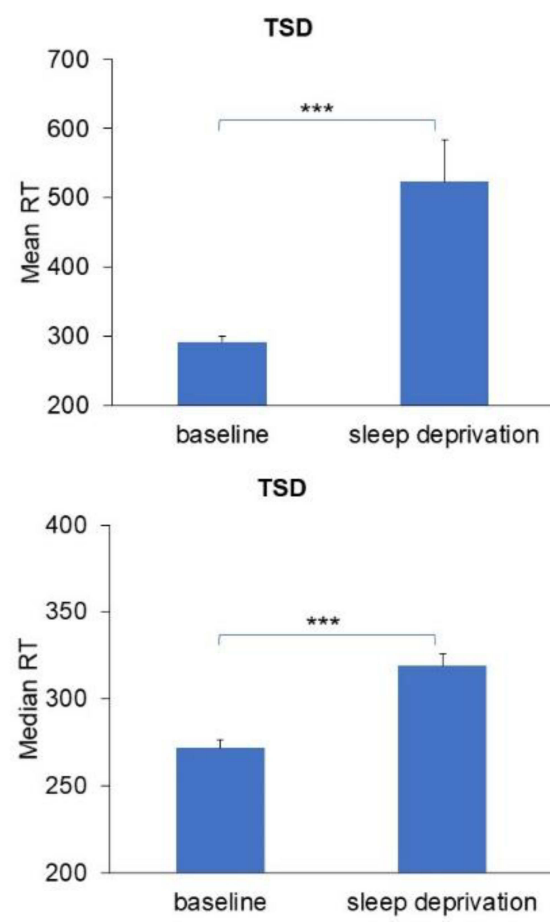

TSD

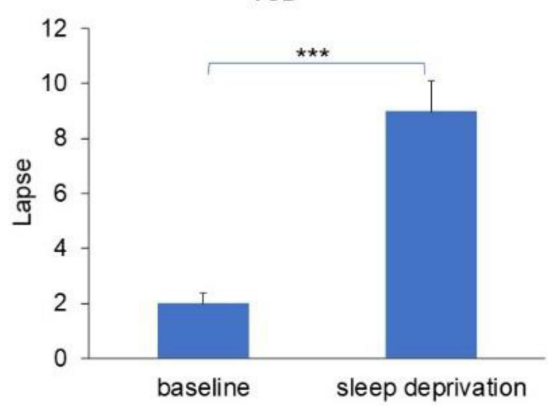

B
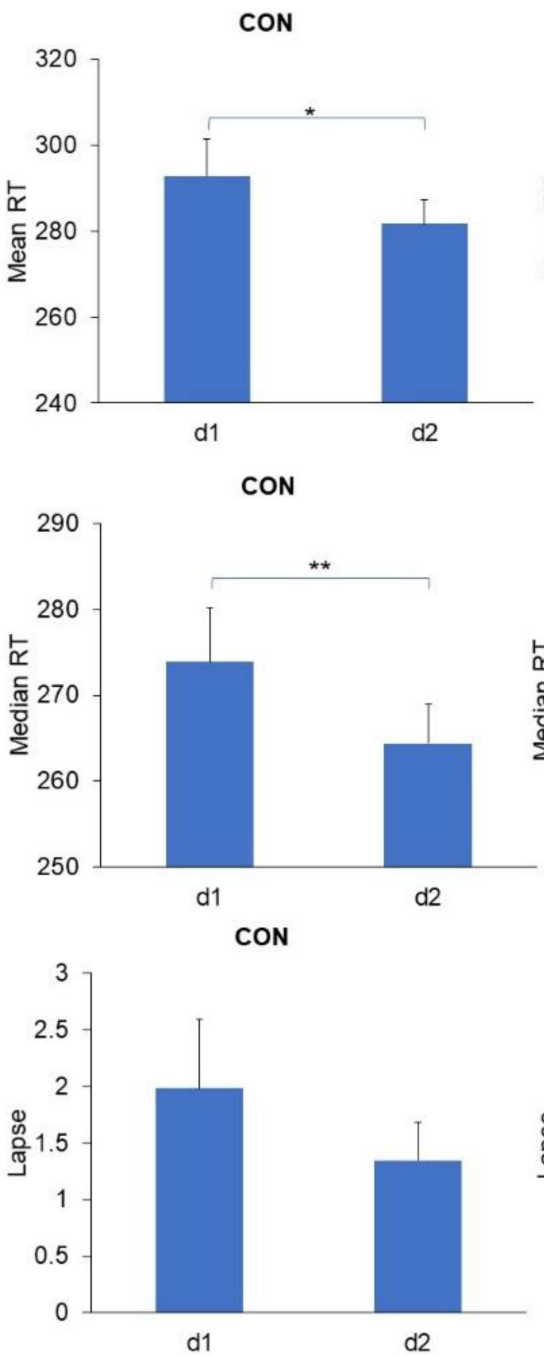

C
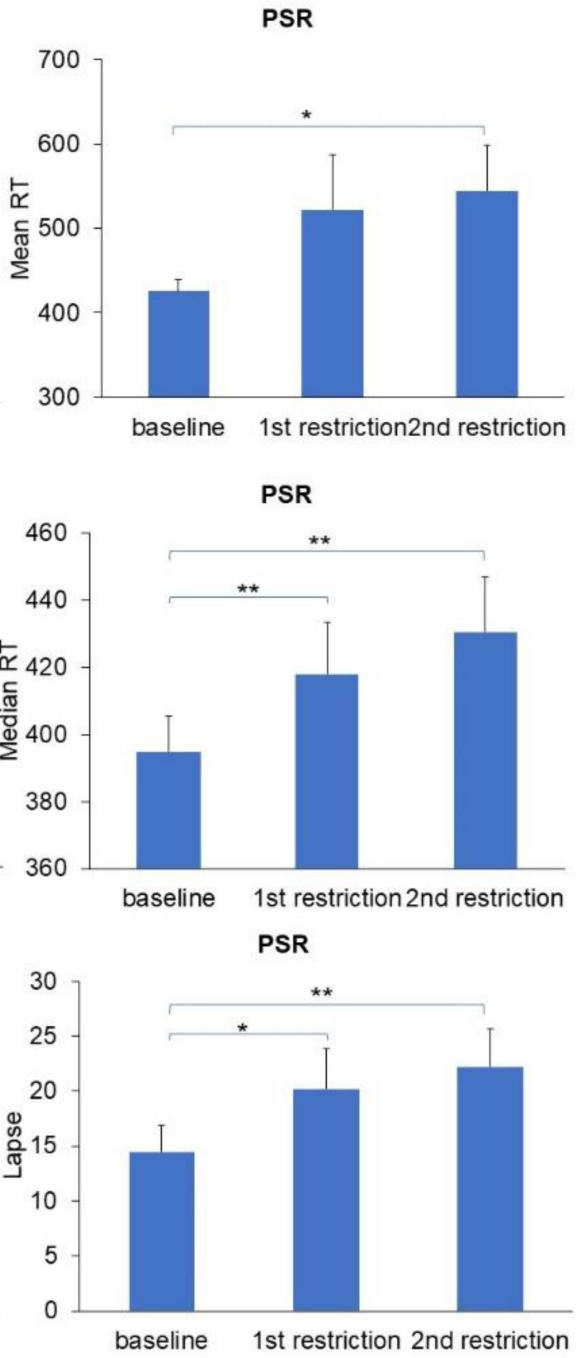

Figure 3 Effects of TSD (Experiment I) and PSR (Experiment 2) on vigilant attention (Mean \pm SE) ((A) PVT performance under TSD condition; (B) PVT performance under CON condition; (C) PVT performance under PSR condition).

Notes: ${ }^{*} p<0.05$; ** $<0.01$; *** $p<0.001$.

Abbreviations: PSR, partial sleep restriction; TSD, total sleep deprivation; CON, normal sleep (the control study); SE, standard error of the mean; dI, day I in control group (baseline); d2, day 2 in control group (control).

the Go/No-Go task $(r=0.35, F D R=0.039)$, and there was a trend of positive correlation between the changes in PVT median RT and the changes in errors of omission on the $\mathrm{Go} /$ No-Go task $(r=0.29, F D R=0.093)$. However, there were no significant associations between changes in the PVT performance and changes in the Go RT and errors of commission of the Go/No-Go task (all $p>0.05$ ). The changes of PVT mean RT explained about $18.0 \%$ of the changes of the Go RT, whereas the changes of PVT median RT and number of lapses explained about $8.4 \%$ and $12.4 \%$ of the changes of errors of omission in the Go/No-Go task, respectively.
The results from correlation analyses of PVT and Go/Go/No-Go task data for the PSR group are shown in Figure 4D and listed in Table 2B. After two nights of PSR, there was also a trend of positive correlation between the changes in PVT median RT and the changes in the Go RT on the Go/No-Go task (Figure 4D, Pearson $\mathrm{r}=0.487, p=0.056$; Spearman rho $=0.400, p=0.125)$. Regression analysis showed that after two nights of PSR, the changes in PVT median RT explained about $23.7 \%$ of the changes in the Go RT on the Go/No-Go task. 
A

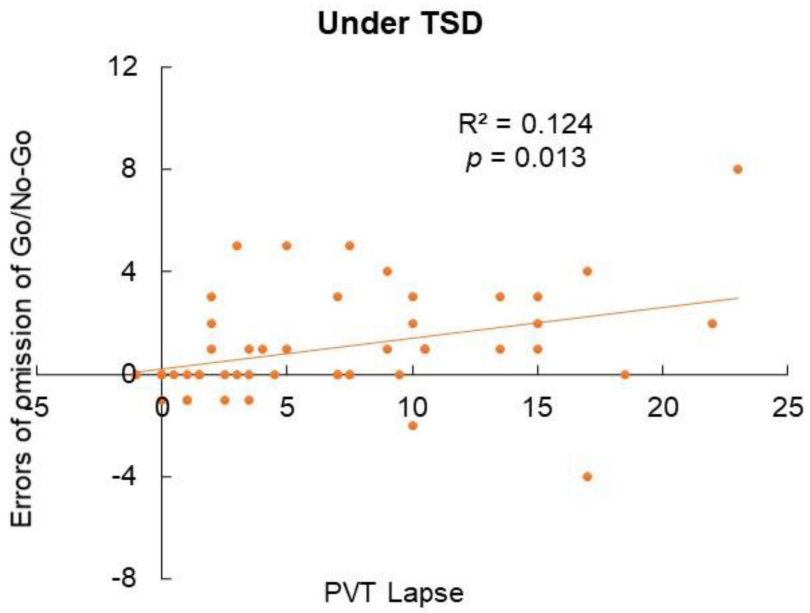

C

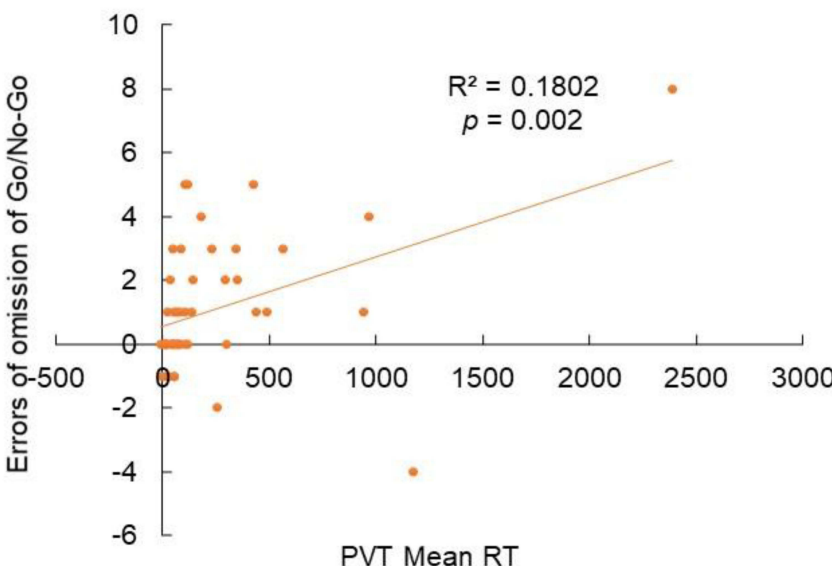

B

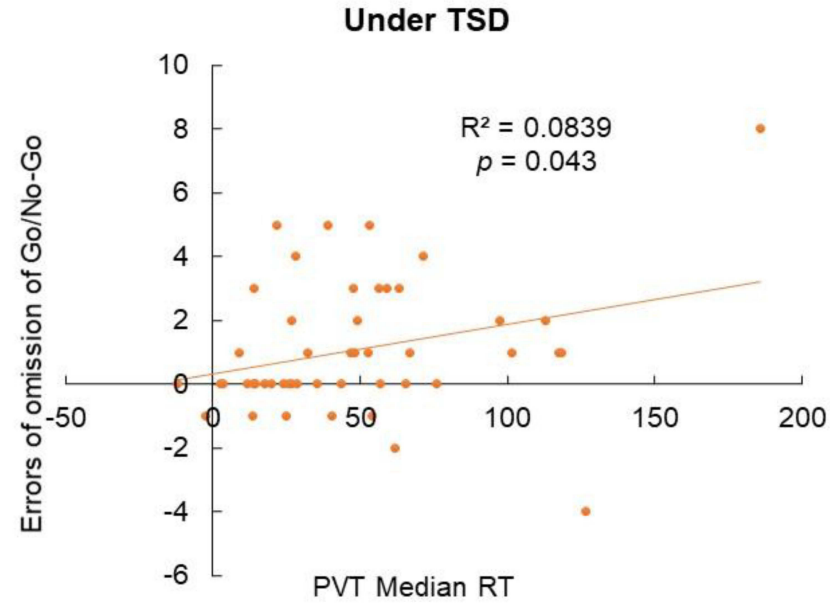

D

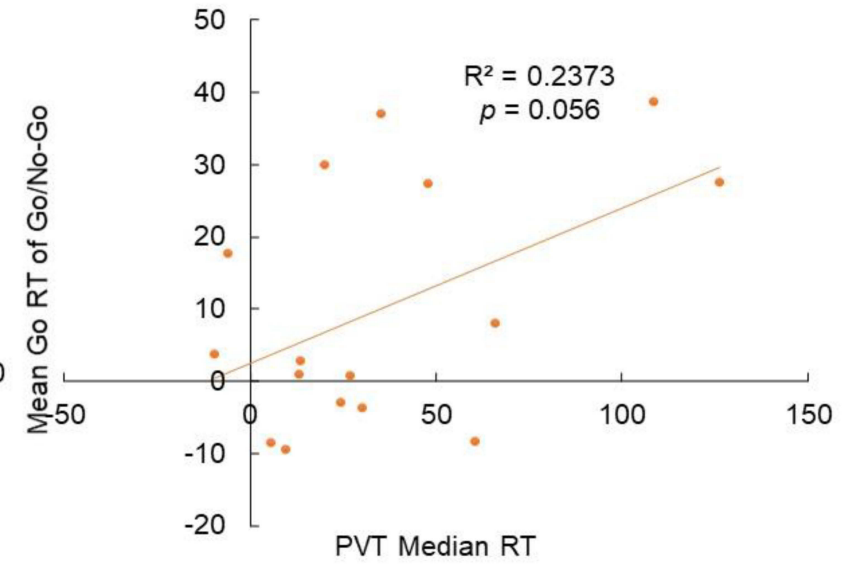

Figure 4 Scatter plot of changes in PVT and Go/No-Go task under TSD (Experiment I) and PSR (Experiment 2) ((A-C) under TSD; (D) under 2nd night of PSR). R $^{2}$ and $P$ values are from regression analyses.

Abbreviations: PSR, partial sleep restriction; TSD, total sleep deprivation; CON, normal sleep (the control study).

\section{Discussion}

This study investigated the effects of both TSD and PSR on inhibition control and further examined whether changes in vigilant attention were associated with the changes in inhibition control after sleep loss. We found that both TSD and PSR negatively affected the levels of inhibition control and the levels of vigilant attention; and that the impairment of inhibition control after sleep loss was significantly correlated with a decline in vigilant attention.

\section{Effects of TSD and PSR on Inhibition Control}

After two nights of PSR or one night of TSD, there were significant increases in both the Go RT and errors of omission in the Go/No-Go task, compared with no significant change in performance seen in the control group. In addition, errors of omission tended to increase after the first night of PSR and further increased after two nights of PSR. This is most likely due to the gradual increase in the accumulation of deficits related to sleep loss as sleep restriction continued. Also, errors of commission did not change significantly after one or two nights of PSR. However, there was a marginal increase after TSD, while an opposite downward trend was observed in the control group. This suggests that changes in errors of commission were unmasked only with higher sleep pressure induced by TSD. In contrast, changes in the control group may be due to the dissipation of existing sleep debt, since a similar trend was also observed in the control group on the PVT results.

The Go RT and errors of omission were affected by both TSD and PSR, but errors of commission were not affected by either sleep condition. This might be explained by the "controlled attention" model, ${ }^{59}$ which suggests that 
Table 2 Pearson Correlation and Spearman Correlation Between the Change in the Go/No-Go Task and PVT After (A) Total Sleep Deprivation and (B) Partial Sleep Restriction

\begin{tabular}{|c|c|c|c|c|}
\hline & \multirow[t]{2}{*}{ Go/No-Go } & \multicolumn{3}{|c|}{ PVT } \\
\hline & & Median RT & Mean RT & Lapses \\
\hline \multicolumn{5}{|l|}{ (A) after TSD } \\
\hline \multirow{9}{*}{$\begin{array}{l}\Delta \text { (total sleep deprivation- } \\
\text { baseline) }\end{array}$} & \multicolumn{4}{|c|}{ Go RT } \\
\hline & Pearson $r$ & 0.183 & 0.077 & 0.254 \\
\hline & Spearman rho & 0.230 & 0.199 & 0.230 \\
\hline & \multicolumn{4}{|c|}{ Errors of omission } \\
\hline & Pearson $r$ & $0.290 *$ & $0.425^{* *}$ & $0.352 *$ \\
\hline & Spearman rho & $0.315^{*}$ & $0.50 I^{* * *}$ & $0.430 * *$ \\
\hline & \multicolumn{4}{|c|}{ Errors of commission } \\
\hline & Pearson $r$ & 0.162 & 0.030 & 0.064 \\
\hline & Spearman rho & 0.161 & 0.105 & 0.083 \\
\hline \multicolumn{5}{|l|}{ (B) after PSR } \\
\hline \multirow{9}{*}{$\begin{array}{l}\Delta \text { (Ist sleep restriction - } \\
\text { baseline) }\end{array}$} & \multicolumn{4}{|c|}{ Go RT } \\
\hline & Pearson $r$ & 0.008 & 0.007 & 0.039 \\
\hline & Spearman rho & 0.034 & $0.24 I$ & 0.143 \\
\hline & \multicolumn{4}{|c|}{ Errors of omission } \\
\hline & Pearson $r$ & 0.421 & $0.45 I^{\#}$ & 0.025 \\
\hline & Spearman rho & 0.178 & -0.050 & -0.006 \\
\hline & \multicolumn{4}{|c|}{ Errors of commission } \\
\hline & Pearson $r$ & 0.189 & -0.237 & 0.150 \\
\hline & Spearman rho & 0.213 & -0.015 & 0.094 \\
\hline \multirow{9}{*}{$\begin{array}{l}\Delta \text { ( } 2 \text { nd sleep restriction - } \\
\text { baseline) }\end{array}$} & \multicolumn{4}{|c|}{ Go RT } \\
\hline & Pearson $r$ & $0.487^{\#}$ & $0.34 I$ & 0.247 \\
\hline & Spearman rho & 0.400 & 0.418 & 0.280 \\
\hline & \multicolumn{4}{|c|}{ Errors of omission } \\
\hline & Pearson $r$ & -0.055 & 0.074 & -0.187 \\
\hline & Spearman rho & -0.094 & -0.059 & -0.001 \\
\hline & \multicolumn{4}{|c|}{ Errors of commission } \\
\hline & Pearson $r$ & -0.036 & -0.001 & 0.167 \\
\hline & Spearman rho & 0.088 & 0.195 & 0.282 \\
\hline
\end{tabular}

Notes: ${ }^{*} p<0.05 ;{ }^{* *} p<0.01 ; *^{* * *} p<0.001 ;{ }^{*} p<0.1$.

Abbreviations: TSD, total sleep deprivation; PSR, partial sleep restriction.

tasks that are monotonous or intrinsically less engaging are more severely affected by sleep deprivation because greater top-down control is required to sustain optimal performance on these tasks. This model has been supported by other studies. ${ }^{60}$ It is possible that the errors of commission may reflect a compensatory effort of the participants in the current study. Specifically, the process of errors of commission was less monotonous and required more controlled attention, leading this measure to be less affected by sleep deprivation.

In addition, the Go stimulus represents response selection, and the No-Go stimulus represents response inhibition. Previous studies suggest that the neural correlates of two processes may have some overlap. ${ }^{61}$ For example, an 
animal study reported that some of the rostral portions of the supplementary motor area (pre-SMA) neurons responded to the Go stimulus (Go type), some neurons responded to the No-Go stimulus (No-Go type), and some neurons responded to both. Further analyses demonstrated that the No-Go and dual-type neurons were activated earlier than the Go neurons, suggesting that the process of reaction selection involves first the inhibition of other reactions and then the selection of new reactions. ${ }^{62}$ Similar findings were also reported in human experiments. ${ }^{63,64}$ Taken together, these studies suggested that the Go stimulus is intended to create a dominant tendency of response, which requires inhibition in the presence of No-Go stimuli. ${ }^{10}$ In this study, the Go RT and errors of omission belong to the process of reaction selection, while errors of commission belong to the process of reaction inhibition. The increase in Go RT and the decrease in the errors of omission indicate the impairment of inhibition control function. However, there was no significant increase in the errors of commission. One possible explanation for this finding is that there may be a practice effect for the No-Go stimulus; similar to what was observed in the control group, in which the errors of commission on the second normal night tended to decrease compared with the first baseline night $(p=0.055)$.

The results of impaired inhibition control after both TSD and PSR are consistent with most previous studies. ${ }^{17-19,24-27,31}$ For example, in a meta-analysis, a total of 15 studies were included to assess the effect of sleep restriction on inhibition control, and the results showed that sleep deprivation had a small to moderate negative impact on inhibition control. ${ }^{14}$ In another study in which 12 participants spent 14 days in the laboratory with 2 baseline days followed by 7 days of partial sleep restriction and 3 recovery days, researchers observed a significant sleep restriction effect on errors of commission in the Go/No-Go task. ${ }^{18}$ However, a few studies have found no significant changes in individuals' levels of inhibition control after TSD or PSR. ${ }^{23,28,41}$ For example, some researchers posit that sleep deprivation does not affect inhibition per se, but rather affects a supervisory attentional process, which is regarded as an active effort to cope with a complex task. ${ }^{65}$ Comparing these studies, we postulate that the inconsistent effects of sleep deprivation on higher cognitive function may be due to confounding factors such as mood, motivation, task difficulty, and complexity. In addition, the final performance result often depends on the interaction of individual characteristics, sensory perception, alertness, working memory, and decision-making, etc. One study found that individuals with longer sleep habits are more susceptible to PSR than those with shorter sleep habits. ${ }^{27}$ In conclusion, we found sleep deprivation negatively impacted the level of inhibition control in this experiment.

\section{Effect of TSD and PSR on Vigilant Attention}

Median RT and the number of lapses on the PVT both increased significantly after one and two nights of PSR and after a night of TSD. A similar result was found for mean RT, which increased significantly after two nights of PSR and one night of TSD. In contrast, in the control group, the median RT and mean RT was significantly reduced after the second night of normal sleep compared to baseline sleep on the first night, while the number of lapses was not significantly changed. This finding is not likely due to practice effects as there are few, if any, practice effects on the PVT. We speculate that the faster reaction time in the control group may be due to two possible reasons. One potential reason may be the dissipation of previous sleep debt during sufficient sleep opportunity, and another one could be that the participants were more comfortable with the environment on the second night than the first night. These results confirm our hypothesis that both TSD and PSR on average impair an individual's level of vigilant attention and are consistent with the results of most previous studies. ${ }^{2,7,8,66-70}$

Overall, the decline in vigilant attention seems to be the most consistent robust result of the negative effects of sleep deprivation. Previous studies demonstrated that performance on attention tasks deteriorates in a dosedependent manner with increased wakefulness, owing to the increasing sleep pressure. ${ }^{67,71}$ Numerous studies have investigated the neural mechanisms by which sleep deprivation affects vigilant attention. ${ }^{68}$ Decreased level of vigilant attention following sleep deprivation has been associated with reductions in fMRI signals in the dorsolateral prefrontal cortex (DLPFC) and intraparietal sulcus. $^{38,72-74}$ Under sleep deprivation, attention performance is usually maintained when thalamus activity increases, and absent when thalamus activity decreases significantly. ${ }^{39,72,75,76}$ Therefore, the thalamus could be an interacting node in the effects of sleep deprivation on vigilant attention. ${ }^{68}$ Other studies have found that after sleep deprivation, the midline anterior and posterior 
cortical regions of the default model network (DMN) cannot be completely disengaged during the attention tasks. $^{74,77,78}$ Thus, instability of the DMN may also be associated with impaired attention caused by sleep deprivation.

\section{Relationship Between Changes in Inhibition Control and Deficits in Vigilant Attention}

The present results showed that changes in PVT performance following TSD or PSR accounted for a portion of the changes in the Go RT and errors of omission of the Go/ No-Go task. This suggests that impairment of vigilant attention after sleep loss partially accounts for the effect of sleep deprivation on inhibition control and confirms, to some extent, the vigilance hypothesis.

We found that the change in errors of commission on the Go/No-Go task was not significantly correlated with the change in any of the PVT indicators. One possible explanation is that the Go RT and errors of omission associated with the change in vigilant attention belong to the response selection process, whereas errors of commission belong to the response inhibition process. Although the two processes overlap to a certain extent, ${ }^{9}$ they are not entirely independent. For instance, one previous study found a significant increase in errors of commission after PSR compared with the control group, while no significant difference was observed in the Go RT. ${ }^{27}$ In another study, researchers measured the reaction time and changes in blood flow when the participant performed the PVT and Go/No-Go tasks. ${ }^{79}$ While the PVT results showed increased blood flow in the left dorsolateral prefrontal cortex with a visual stimulus compared to an auditory stimulus, Go/No-Go task results suggested that, compared with auditory stimuli, the blood flow associated with visual stimuli increased on both sides of the inferior temporal gyrus and the right side of the inferior frontal gyrus. ${ }^{79}$ These findings suggest that inhibition control may be related to factors other than vigilance and processing speed.

Another potential explanation may be due to the speed/ accuracy trade-off. The results indicate that there was no significant reduction in the commission errors after TSD or PSR in the present study, but the Go RT was progressively slower. This is consistent with the theory of the speed/ accuracy trade-off. ${ }^{32,80,81}$ That is, participants were able to maintain the ability to withhold response to inhibition trails on the Go/No-Go task by slowing down throughout the task to maintain accuracy. In one previous study, thirteen participants completed a modified version of the PVT (Stop-PVT, measuring both response inhibition and sustained attention) at baseline and $\mathrm{TSD}^{80}$ The results demonstrated that accuracy was not reduced during TSD. However, response times were significantly slower. ${ }^{80}$ In another study, eighteen subjects used a visual search task and PVT measurement in a shift work simulation protocol. ${ }^{81}$ The study results showed that the nighttime decrease in visual search sensitivity was most pronounced on the first night compared with subsequent nights. This was accompanied by a trend towards selective attention becoming "fast and sloppy". ${ }^{81}$

In addition, there may be other pathways underlying the effects of sleep deprivation on inhibition control besides vigilant attention. First, while the PVT involves only one stimulus, the Go/No-Go task involves at least two stimuli. Some studies have used a Go/No-Go task that involves 26 letters, ${ }^{27}$ which inevitably uses working memory. Moreover, while the PVT requires participants to press a button immediately after seeing the stimulus, with no process of choice, the Go/No-Go task asks a participant to press the key only to the Go stimulus and not to the No-Go stimulus, which forces them to decide whether to press the button or not when a stimulus is present. As a result, the Go/No-Go task has a decision-making component inherent to it, which is absent in the PVT. Finally, inhibition control may be less sensitive to sleep changes than vigilant attention. For instance, one study demonstrated that six nights of sleep extension before sleep deprivation did not reduce inhibition control deficits during a total sleep deprivation period. ${ }^{18}$ By contrast, another study found that six nights of sleep extension was sufficient to reduce vigilant attention deficits related to total sleep deprivation. ${ }^{82}$ Therefore, the effects of sleep deprivation on inhibition control likely depend on the interaction of vigilant attention, working memory, decision making, and sensory perception, among other factors.

\section{Limitations and Future Studies}

Several limitations need to be considered for this study. First, the sample size of the PSR experiment was relatively small, which may affect the validity of the PSR conclusions to some extent. Second, we did not explore the neural mechanisms underlying the effects of sleep loss on inhibition control. Previous studies showed that sleep 
deprivation affects different tasks to different degrees, but the general principles behind these differences are not well understood. Some researchers found that, as was true for tasks of working memory and selective attention, DMN activity in the cortical regions of the episodic memory coding task was significantly different after sleep deprivation. ${ }^{83}$ Thus, the unstable control of DMN may be a common neurological deficit in the sleep-deprived human brain for a variety of cognitive tasks, including inhibition control. In addition, as the most severely impaired tests are those known to involve the prefrontal cortex, such as working memory tasks or executive function, some researchers have proposed the prefrontal cortex vulnerability hypothesis. ${ }^{22}$ On the other hand, a previous ERP study found that poor sleep specifically affected the intensity of pre-motor inhibitory processes (NoGo-N2 amplitude), the speed to inhibit a motor response (NoGoP3 latency), and the intensity of task-relevant information processing (Go-P3 amplitude). ${ }^{15,84}$ Additional study showed there was a correlation between the NoGo-P3 and the final process of stopping an already partly activated but inadequate response. ${ }^{33}$ Future studies should explore this question to better understand the neural mechanisms underlying inhibition control after sleep deprivation.

Future studies should consider potential individual differences when studying the effects of sleep deprivation. For instance, one study found that the decline in alertness after sleep deprivation was influenced by different incentives, such that the more rewards an individual received, the less his or her attention level dropped after sleep deprivation. ${ }^{69}$ This suggests that the impact of sleep loss may be at least partially mitigated with sufficient motivation. Another study explored the blood oxygen level-dependent (BOLD) activation both in older adults and younger adults during the Go/ No-Go task following a total of 36 hours of sleep deprivation and found that older adults reported greater BOLD activation compared to young adults. ${ }^{17}$ In addition, Grant et al found that Brain Derived Neurotrophic Factor $(B D N F)$ Met allele carriers were more vulnerable to the effect of sleep deprivation on inhibition control when using the Stroop Color Naming Task as the measurement. ${ }^{85}$ Researchers also found that the adverse effects of sleep deprivation on inhibition control were more pronounced in individuals who spent more habitual time in bed at baseline. $^{22}$ Additionally, tolerance or susceptibility to performance deficits following sleep loss may be associated with certain individual characteristics. ${ }^{86}$

\section{Conclusion}

In summary, both TSD and PSR impaired inhibition control during the Go/No-Go task and vigilant attention during the PVT. The decline in inhibition control following both forms of sleep loss was partly accounted for by impairment in vigilant attention. Findings from the present study support the vigilance hypothesis and raise the possibility that there may be other pathways underlying the effect of sleep deprivation on inhibition control besides vigilant attention.

\section{Acknowledgments}

This research was supported in part by the grants from National Institutes of Health (R01-HL102119, R01MH107571, R21-AG051981, and CTRC UL1RR024134), Shanghai International Studies University Research Projects (Grant number 20171140020), Shanghai Post-doctoral Excellence Program (Grant number 2020367), China Postdoctoral Science Foundation (Grant number 2021M692150), National Natural Science Foundation of China (Grant Number 71942003), and South China Normal University. The funders had no role in the study design, data collection, and analysis, data interpretation, writing of the manuscript, or the decision to submit the article for publication.

\section{Disclosure}

Dr. Tianxin Mao reports grants from South China Normal University, during the conduct of the study. Dr. Olga Galli is an employee of Jazz Pharmaceuticals. The authors report no other conflicts of interest in this work.

\section{References}

1. Akerstedt T. Altered sleep/wake patterns and mental performance. Physiol Behav. 2007;90(2-3):209-218. doi:10.1016/j. physbeh.2006.09.007

2. Dennis LE, Wohl RJ, Selame LA, Goel N. Healthy adults display long-term trait-like neurobehavioral resilience and vulnerability to sleep loss. Sci Rep. 2017;7(1):14889. doi:10.1038/s41598-017-14006-7

3. McHill AW, Hull JT, Cohen DA, et al. Chronic sleep restriction greatly magnifies performance decrements immediately after awakening. Sleep. 2019;42(5):zsz032. doi:10.1093/sleep/zsz032

4. Stojanoski B, Benoit A, Berg VDN, et al. Sustained vigilance is negatively affected by mild and acute sleep loss reflected by reduced capacity for decision making, motor preparation, and execution. Sleep. 2019;42(1):zsy200. doi:10.1093/sleep/zsy200

5. Fobian AD, Rouse J, Stager LM, Long D, Schwebel DC, Avis KT. The effects of sleep deprivation and text messaging on pedestrian safety in university students. Sleep. 2020;43(9):zsaa057. doi:10.1093/sleep/ zsaa057

6. Lehouillier IS, Saksvik SB, Dahlberg J, et al. Mild to moderate partial sleep deprivation is associated with increased impulsivity and decreased positive affect in young adults. Sleep. 2020;43(10): zsaa078. doi:10.1093/sleep/zsaa078 
7. Yamazaki EM, Goel N. Robust stability of trait-like vulnerability or resilience to common types of sleep deprivation in a large sample of adults. Sleep. 2020;43(6):zsz292. doi:10.1093/sleep/zsz292

8. Yamazaki EM, Antler CA, Lasek CR, Goel N. Residual, differential neurobehavioral deficits linger after multiple recovery nights following chronic sleep restriction or acute total sleep deprivation. Sleep. 2021;44(4):zsaa224. doi:10.1093/sleep/zsaa224

9. Simmonds DJ, Pekar JJ, Mostofsky SH. Meta-analysis of Go/No-go tasks demonstrating that fMRI activation associated with response inhibition is task dependent. Neuropsychologia. 2008;46(1):224-232. doi:10.1016/j.neuropsychologia.2007.07.015

10. Congdon E, Mumford JA, Cohen JR, Galvan A, Canli T, Poldrack RA. Measurement and reliability of response inhibition. Front Psychol. 2012;3:37. doi:10.3389/fpsyg.2012.00037

11. Fuster JM. The Prefrontal Cortex. London: Academic Press; 2008.

12. Bari A, Robbins TW. Inhibition and impulsivity: behavioral and neural basis of response control. Prog Neurobiol. 2013;108:44-79. doi:10.1016/j.pneurobio.2013.06.005

13. Donders FC. On the speed of mental processes. Acta Psychol. 1969;30:412-431. doi:10.1016/0001-6918(69)90065-1

14. Lowe CJ, Safari A, Hall PA. The neurocognitive consequences of sleep restriction: a meta-analytic review. Neurosci Biobehav Rev. 2017;80:586-604. doi:10.1016/j.neubiorev.2017.07.010

15. Breimhorst M, Falkenstein M, Marks A, Griefahn B. The relationship between poor sleep and inhibitory functions indicated by event-related potentials. Exp Brain Res. 2008;187(4):631-639. doi:10.1007/s00221-008-1333-9

16. Harrington M, Ashton J, Sankarasubramanian S, Anderson MC, Cairney SA. Losing control: sleep deprivation impairs the suppression of unwanted thoughts. Sleep Med. 2021;9(1):97-113. doi:10.1177/2167702620951511

17. Almklov EL, Drummond SPA, Orff H, Alhassoon OM. The effects of sleep deprivation on brain functioning in older adults. Behav Sleep Med. 2014;12(1):324-345. doi:10.1080/15402002.2014.905474

18. Rabat A, Arnal PJ, Monnard H, et al. Limited benefit of sleep extension on cognitive deficits during total sleep deprivation: illustration with two executive processes. Front Neurosci. 2019;13:591. doi:10.3389/fnins.2019.00591

19. Anderson C, Platten CR. Sleep deprivation lowers inhibition and enhances impulsivity to negative stimuli. Behav Brain Res. 2011;217(2):463-466. doi:10.1016/j.bbr.2010.09.020

20. Bocca ML, Marie S, Chavoix C. Impaired inhibition after total sleep deprivation using an antisaccade task when controlling for circadian modulation of performance. Physiol Behav. 2014;124:123-128. doi:10.1016/j.physbeh.2013.10.024

21. Drummond SP, Paulus MP, Tapert SF. Effects of two nights sleep deprivation and two nights recovery sleep on response inhibition. $J \quad$ Sleep Res. 2006;15(3):261-265. doi:10.1111/j.13652869.2006.00535.x

22. Harrison Y, Horne JA, Rothwell A. Prefrontal neuropsychological effects of sleep deprivation in young adults - a model for healthy aging? Sleep. 2000;23:1067-1073. doi:10.1093/sleep/23.8.1f

23. Patrick Y, Lee A, Raha O, et al. Effects of sleep deprivation on cognitive and physical performance in university students. Sleep Biol Rhythms. 2017;15(3):217-225. doi:10.1007/s41105-017-0099-5

24. Cohen-Zion M, Shabi A, Levy S, Glasner L, Wiener A. Effects of partial sleep deprivation on information processing speed in adolescence. $J$ Int Neuropsychol Soc. 2016;22(4):388-398. doi: $10.1017 / \mathrm{S} 1355617716000072$

25. Stenuit P, Kerkhofs M. Effects of sleep restriction on cognition in women. Biol Psychol. 2008;77(1):81-88. doi:10.1016/j. biopsycho.2007.09.011

26. Jarraya M, Jarraya S, Chtourou H, Souissi N, Chamari K. The effect of partial sleep deprivation on the reaction time and the attentional capacities of the handball goalkeeper. Biol Rhythm Res. 2012;44:503-510. doi:10.1080/09291016.2012.721589
27. Demos KE, Hart CN, Sweet LH, et al. Partial sleep deprivation impacts impulsive action but not impulsive decision-making. Physiol Behav. 2016;164(Part A):214-219. doi:10.1016/j. physbeh.2016.06.003

28. Rossa KR, Smith SS, Allan AC, Sullivan KA. The effects of sleep restriction on executive inhibitory control and affect in young adults. $J \quad$ Adolesc Health. 2014;55(2):287-292. doi:10.1016/j. jadohealth.2013.12.034

29. Schumacher AM, Miller AL, Watamura SE, Kurth S, Lebourgeois MK. Sleep moderates the association between response inhibition and self-regulation in early childhood. J Clin Child Adolesc Psychol. 2017;46(2):222-235. doi:10.1080/15374416.2016.1204921

30. Cerolini S, Ballesio A, Ferlazzo F, Lucidi F, Lombardo C. Decreased inhibitory control after partial sleep deprivation in individuals reporting binge eating: preliminary findings. PeerJ. 2020;8(5):e9252. doi: 10.7717 peerj. 9252

31. Rabat A, Gomez-Merino D, Roca-Paixao L, et al. Differential kinetics in alteration and recovery of cognitive processes from a chronic sleep restriction in young healthy men. Front Behav Neurosci. 2016;10:95. doi:10.3389/fnbeh.2016.00095

32. Gosselin A, De Koninck J, Campbell KB. Disentangling specific inhibitory versus general decision-making processes during sleep deprivation using a Go/No Go ERP paradigm. Int J Psychophysiol. 2019;141:18-27. doi:10.1016/j.ijpsycho.2019.04.007

33. Schapkin SA, Falkenstein M, Marks A, et al. After effects of noise-induced sleep disturbances on inhibitory functions. Life Sci. 2006;78(10):1135-1142. doi:10.1016/j.lfs.2005.06.048

34. Alhola P, Polo-Kantola P. Sleep deprivation: impact on cognitive performance. Neuropsychiatr Dis Treat. 2007;3(5):553-567.

35. Goel N, Rao H, Durmer JS, Dinges DF. Neurocognitive consequences of sleep deprivation. Semin Neurol. 2009;29:320-339. doi:10.1055/s-0029-1237117

36. Goel N, Basner M, Rao H, Dinges DF. Circadian rhythms, sleep deprivation, and human performance. Prog Mol Biol Transl Sci. 2013;119:155-190. doi:10.1016/B978-0-12-396971-2.00007-5

37. Lim J, Dinges DF. Sleep deprivation and vigilant attention. Ann N Y Acad Sci. 2008;1129:305-322. doi:10.1196/annals.1417.002

38. Chee MW, Goh CS, Namburi P, Parimal S, Seidl KN, Kastner S. Effects of sleep deprivation on cortical activation during directed attention in the absence and presence of visual stimuli. Neuroimage. 2011;58(2):595-604. doi:10.1016/j.neuroimage.2011.06.058

39. Chee MW, Tan JC, Parimal S, Zagorodnov V. Sleep deprivation and its effects on object-selective attention. Neuroimage. 2010;49 (2):1903-1910. doi:10.1016/j.neuroimage.2009.08.067

40. Lim J, Dinges DF. A meta-analysis of the impact of short-term sleep deprivation on cognitive variables. Psychol Bull. 2010;136 (3):375-389. doi:10.1037/a0018883

41. Sturm W, De Simone A, Krause BJ, et al. Functional anatomy of intrinsic alertness: evidence for a fronto-parietal-thalamic-brainstem network in the right hemisphere. Neuropsychologia. 1999;37 (7):797-805. doi:10.1016/S0028-3932(98)00141-9

42. Sturm W, Willmes K. On the functional neuroanatomy of intrinsic and phasic alertness. Neuroimage. 2001;14(1):76-84. doi:10.1006/ nimg.2001.0839

43. Smith CS, Reilly C, Midkiff K. Evaluation of three circadian rhythm questionnaires with suggestions for an improved measure of morningness. J Appl Psychol. 1989;74(5):728-738. doi:10.1037/ 0021-9010.74.5.728

44. Yang FN, Xu S, Chai Y, Basner M, Dinges DF, Rao H. Sleep deprivation enhances inter-stimulus interval effect on vigilant attention performance. Sleep. 2018;41(12):1-12. doi:10.1093/sleep/zsy189

45. Chai Y, Fang Z, Yang FN, et al. Two nights of recovery sleep restores hippocampal connectivity but not episodic memory after total sleep deprivation. Sci Rep. 2020;10(1):8774. doi:10.1038/s41598-02065086-x 
46. Dinges DF, Powell JW. Microcomputer analyses of performance on a portable, simple visual RT task during sustained operations. Behav Res Methods Instrum Comput. 1985;17(6):652-655. doi:10.3758/ BF03200977

47. Basner M, Hermosillo E, Nasrini J, et al. Repeated administration effects on psychomotor vigilance test (PVT) performance. Sleep. 2018;41(1):1-6. doi:10.1093/sleep/zsx187

48. Horne JA, Ostberg OA. A self-assessment questionnaire to determine morningness-eveningness in human circadian rhythms. Int J Chronobiol. 1976;4(2):97-110.

49. Zhang B, Hao YL, Rong RG. The reliability and validity of Chinese version morningness /eveningness questionnaire. Chin J Behavioral Med Sci. 2006;15(9):856-858.

50. Buysse DJ, Reynolds CF, Monk TH, Berman SR, Kupfer DJ. The Pittsburgh Sleep Quality Index: a new instrument for psychiatric practice and research. Psychiatry Res. 1989;28:193-213. doi:10.1016/0165-1781(89)90047-4

51. Buysse DJ, Reynolds CF, Monk TH, Hoch CC, Yeager AL, Kupfer DJ. Quantification of subjective sleep quality in healthy elderly men and women using the Pittsburgh Sleep Quality Index. J Sleep Res Sleep Med. 1991;14(4):331-338. doi:10.1093/sleep/ 14.4.331

52. Liu XC, Tang MQ, Hu L, et al. Reliability and validity of the Pittsburgh sleep quality index. Chin $J$ Psychiatry. 1996;29 (2):103-107.

53. Zung WWK. A rating instrument for anxiety disorders. Psychosomatics. 1971;12:371-379. doi:10.1016/S0033-3182(71) 71479-0

54. Wu WY. Self-rating anxiety scale. Shanghai Arch Psychiatry. 1990;2:44.

55. Goldberg DP. The Detection of Psychiatric Illness by Questionnaire. Oxford: Oxford University Press; 1972.

56. Li H, Mei JR. Assessing psychological well-being of college student: psychometric properties of GHQ-20. Psychol Dev Educ. 2002;18 (1):75-79.

57. Beck AT, Beamesberfer A. Assessment of depression: the depression inventory. Psychol Meas Psychopharmacol. 1974;7:151-169. doi:10.1159/000395074

58. Benjamini Y, Hochberg Y. Controlling the false discovery rate: a practical and powerful approach to multiple testing. J R Stat Soc Series B Stat Methodol. 1995;57(1):289-300. doi:10.1111/j.25176161.1995.tb02031.x

59. Pilcher JJ, Band D, Odle-Dusseau HN, Muth ER. Human performance under sustained operations and acute sleep deprivation conditions: toward a model of controlled attention. Aviat Space Environ Med. 2007;78(Suppl 5):B15-B24.

60. Kusztor A, Raud L, Juel BE, Nilsen AS, Storm JF, Huster RJ. Sleep deprivation differentially affects subcomponents of cognitive control. Sleep. 2019;42(4):zsz016. doi:10.1093/sleep/zsz016

61. Mostofsky SH, Simmonds DJ. Response inhibition and response selection: two sides of the same coin. J Cogn Neurosci. 2008;20 (5):751-761. doi:10.1162/jocn.2008.20500

62. Isoda M, Hikosaka O. Switching from automatic to controlled action by monkey medial frontal cortex. Nat Neurosci. 2007;10(2):240-248. doi:10.1038/nn1830

63. Braver TS, Barch DM, Gray JR, Molfese DL, Snyder A. Anterior cingulate cortex and response conflict: effects of frequency, inhibition and errors. Cerebral Cortex. 2001;11(9):825-836. doi:10.1093/cercor/11.9.825

64. Aron AR, Poldrack RA. The cognitive neuroscience of response inhibition: relevance for genetic research in attention-deficit/hyperactivity disorder. Biol Psychiatry. 2005;57(11):1285-1292. doi:10.1016/j.biopsych.2004.10.026

65. Jennings JR, Monk TH, van der Molen MW. Sleep deprivation influences some but not all processes of supervisory attention. Psychol Sci. 2003;14:473-479. doi:10.1111/1467-9280.02456
66. Banks S, Van Dongen HPA, Maislin G, Dinges DF. Neurobehavioral dynamics following chronic sleep restriction: dose-response effects of one night for recovery. Sleep. 2010;33(8):1013-1026. doi:10.1093/ sleep/33.8.1013

67. Belenky G, Wesensten NJ, Thorne DR, et al. Patterns of performance degradation and restoration during sleep restriction and subsequent recovery: a sleep dose-response study. J Sleep Res. 2003;12(1):1-12. doi:10.1046/j.1365-2869.2003.00337.x

68. Krause AJ, Simon EB, Mander BA, et al. The sleep-deprived human brain. Nat Rev Neurosci. 2017;18:404-418. doi:10.1038/nrn.2017.55

69. Massar S, Lim J, Sasmita K, Chee M. Sleep deprivation increases the costs of attentional effort: performance, preference and pupil size. Neuropsychologia. 2019;123:169-177. doi:10.1016/j. neuropsychologia.2018.03.032

70. Short MA, Weber N, Reynolds C, Coussens S, Carskadon MA. Estimating adolescent sleep need using dose-response modeling. Sleep. 2018;41(4):zsy011. doi:10.1093/sleep/zsy011

71. Van Dongen HP, Maislin G, Mullington JM, Dinges DF. The cumulative cost of additional wakefulness: dose-response effects on neurobehavioral functions and sleep physiology from chronic sleep restriction and total sleep deprivation. Sleep. 2003;26:117-126. doi:10.1093/sleep/26.2.117

72. Chee MW, Tan JC, Zheng H, Parimal S, Weissman DH, Zagorodnov V. Lapsing during sleep deprivation is associated with distributed changes in brain activation. $J$ Neurosci. 2008;28 (21):5519-5528. doi:10.1523/JNEUROSCI.0733-08.2008

73. Chee MW, Tan JC. Lapsing when sleep deprived, neural activation characteristics of resistant and vulnerable individuals. Neuroimage. 2010;51(2):835-843. doi:10.1016/j.neuroimage.2010.02.031

74. Czisch M, Wehrle R, Harsay HA, et al. On the need of objective vigilance monitoring, effects of sleep loss on target detection and task-negative activity using combined EEG/fMRI. Front Neurol. 2012;3(67):1-12. doi:10.3389/fneur.2012.00067

75. Durmer JS, Dinges DF. Neurocognitive consequences of sleep deprivation. Semin Neurol. 2005;25:117-129. doi:10.1055/s-00291237117

76. Thomas M, Sing H, Belenky G, et al. Neural basis of alertness and cognitive performance impairments during sleepiness I. Effects of 24 $\mathrm{h}$ of sleep deprivation on waking human regional brain activity. J Sleep Res. 2000;9(4):335-352. doi:10.1046/j.1365-2869.2000.00225.x

77. Tomasi D, Wang RL, Telang F, et al. Impairment of attentional networks after 1 night of sleep deprivation. Cerebral Cortex. 2009;19(1):233-240. doi:10.1093/cercor/bhn073

78. Drummond SPA, Bischoff-Grethe A, Dinges DF, Ayalon L, Mednick S, Meloy MJ. The neural basis of the psychomotor vigilance task. Sleep. 2005;28(9):1059-1068. doi:10.1093/sleep/28.9.1059

79. Hiroyasu T, Fukushima A, Yokouchi H. Differences in blood flow between auditory and visual stimuli in the Psychomotor Vigilance Task and GO/NO GO Task. In: 2012 Annual International Conference of the IEEE Engineering in Medicine and Biology Society; 2012; IEEE. 1466-1469. doi:10.1109/EMBC.2012.6346217

80. Hudson AN, Hansen DA, Hinson JM, et al. Speed/accuracy trade-off in the effects of acute total sleep deprivation on a sustained attention and response inhibition task. Chronobiol Int. 2020;37(910):1441-1444. doi:10.1080/07420528.2020.1811718

81. Santhi N, Horowitz TS, Duffy JF, Czeisler CA. Acute sleep deprivation and circadian misalignment associated with transition onto the first night of work impairs visual selective attention. PLoS One. 2007;2(11):e1233. doi:10.1371/journal.pone.0001233

82. Arnal PJ, Sauvet F, Leger D, et al. Benefits of sleep extension on sustained attention and sleep pressure before and during total sleep deprivation and recovery. Sleep. 2015;38:1935-1943. doi:10.5665/ sleep. 5244

83. Gujar N, Yoo SS, Hu P, Walker MP. The unrested resting brain: sleep deprivation alters activity within the default-mode network. $J$ Cogn Neurosci. 2010;22:1637-1648. doi:10.1162/jocn.2009.21331 
84. Burle B, Vidal F, Bonnet M. Electroencephalographic Nogo potentials in a no-movement context: the case of motor imagery in humans. Neurosci Lett. 2004;360:77-80. doi:10.1016/j.neulet.2004.02.034

85. Grant LK, Cain SW, Chang AM, Saxena R, Czeisler CA, Anderson C. Impaired cognitive flexibility during sleep deprivation among carriers of the Brain Derived Neurotrophic Factor (BDNF) Val66Met allele. Behav Brain Res. 2018;2017:51-55. doi:10.1016/j. bbr.2017.09.025
86. Van Dongen HPA, Baynard MD, Maislin G, Dinges DF. Systematic interindividual differences in neurobehavioral impairment from sleep loss: evidence of trait-like differential vulnerability. Sleep. 2004;27 (3):423-433. doi:10.1093/sleep/27.3.423

\section{Publish your work in this journal}

Nature and Science of Sleep is an international, peer-reviewed, open access journal covering all aspects of sleep science and sleep medicine, including the neurophysiology and functions of sleep, the genetics of sleep, sleep and society, biological rhythms, dreaming, sleep disorders and therapy, and strategies to optimize healthy sleep.
The manuscript management system is completely online and includes a very quick and fair peer-review system, which is all easy to use. Visit http://www.dovepress.com/testimonials.php to read real quotes from published authors. 